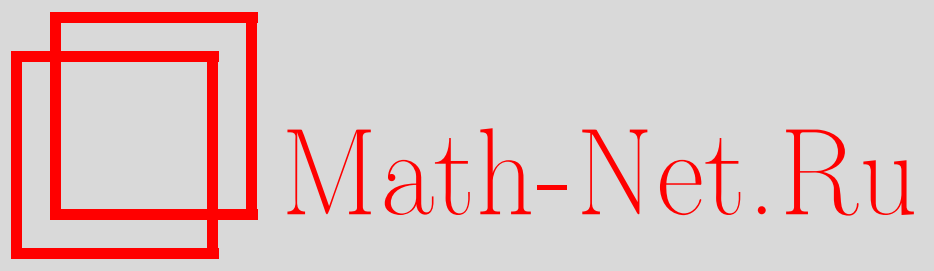

А. В. Иванов, Диаграмматика теплового ядра ковариантного оператора Лапласа, ТМФ, 2019, том 198, номер 1, 113-132

DOI: https://doi.org/10.4213/tmf9515

Использование Общероссийского математического портала Math-Net.Ru подразумевает, что вы прочитали и согласны с пользовательским соглашением http://www . mathnet.ru/rus/agreement

Параметры загрузки:

IP: 18.209 .158 .208

26 апреля 2023 г., 15:20:23

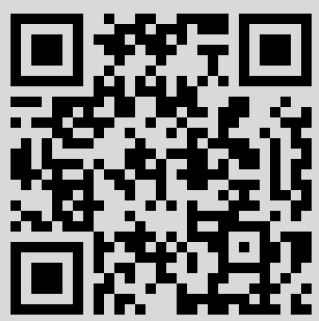




\title{
ДИАГРАММАТИКА ТЕПЛОВОГО ЯДРА КОВАРИАНТНОГО ОПЕРАТОРА ЛАПЛАСА
}

\begin{abstract}
Приводится диаграммная техника, с помощью которой вычисляются коэффициенты Сили-Девитта в случае ковариантного оператора Лапласа. На основе комбинаторных свойств коэффициентов строится матричный формализм и выводится формула для произвольного коэффициента.
\end{abstract}

Ключевые слова: тепловое ядро, коэффициенты Сили-Девитта, калибровочная связность, диаграммная техника, определитель оператора, ковариантный оператор Лапласа, теория Янга-Миллса.

DOI: https://doi.org/10.4213/tmf9515

\section{1. ВВЕДЕНИЕ}

Мотивировка исследований. Изначально метод собственного времени Фока, описанный в статье [1], использовался для работы с функциями Грина, однако затем он нашел свое применение к вопросам калибровочной инвариантности [2] и к квантовой гравитации [3]. В настоящее время данный подход является достаточно удобным инструментом в математической [4] и особенно теоретической физике, который используется для петлевых расчетов, поиска расходимостей и перенормировки. В качестве примера можно привести подсчет двух и трех петель для теории Янга-Миллса в формализме фонового поля [5], [6]. Подсчет коэффициентов разложения теплового ядра по степеням собственного времени (коэффициенты Сили-Девитта) является отдельной трудоемкой задачей. Так, в случае дифференциального оператора второго порядка получены ответы вплоть до степени $\tau^{5-d / 2}$, где $d$ - размерность пространства [7]-[11]. Наиболее общий обзор на данную тему можно найти в работе [12].

Основным объектом настоящего исследования является ковариантный оператор Лапласа в пространстве с плоской метрикой. В рамках общей теории рассматривается его тепловое ядро, коэффициенты которого при разложении в ряд по собственному времени подчиняются соотношениям (11). Эти дифференциальные уравнения

Работа выполнена при поддержке Российского научного фонда (проект № 14-11-00598).

* Санкт-Петербургское отделение Математического института им. В. А. Стеклова Российской академии наук, Санкт-Петербург, Россия. E-mail: regul1@mail.ru 
допускают решения (14) и (25), однако при подсчете следа такая форма решений не является удобной. Основная цель статьи состоит в изучении комбинаторных свойств коэффициентов Сили-Девитта на основе построенной диаграммной техники. Данная работа является продолжением и математическим обоснованием формализма, предложенного в [13].

Наиболее близкой к предлагаемому подходу является ковариантная теория возмущений [14], в которой также используется разложение операторной экспоненты. Однако авторы работ [14] не интересовались комбинаторикой высших порядков теории возмущений. Высокие порядки разложения теплового ядра были получены в статье [15] при определенных ограничениях на форму напряженности калибровочного поля. В настоящей работе такие ограничения не налагаются.

Результаты работы. Статья состоит из двух основных частей. Основным результатом первой части является построение диаграммной техники для работы с тепловым ядром в случае ковариантного оператора Лапласа. В определениях 2-4 вводятся основные понятия данной диаграмматики. Также доказана теорема о дифференцировании диаграммы, играющая ключевую роль в вычислениях. Затем в качестве примера получены первые коэффициенты, возникающие при работе с детерминантом в теории Янга-Миллса [16], [17]. Вторая часть статьи посвящена выводу формулы для произвольного коэффициента Сили-Девитта $a_{n}(x, x)$ с использованием матриц и операторов, которые действуют на матрицы. Основной результат содержится в формулах (44), (46) и (52). В заключение приводится расчет коэффициента $a_{3}(x, x)$ при степени $\tau^{3-d / 2}$.

\section{2. ТЕПЛОВОЕ ЯДРО}

Фундаментальное решение для оператора $A_{0}=-\partial^{\mu} \partial_{\mu}$ удовлетворяет уравнению

$$
A_{0} G_{0}(x, y)=\delta(x-y) \text {. }
$$

Согласно методу теплового ядра [1] для нахождения $G_{0}(x, y)$ необходимо найти функцию $K_{0}(x, y ; \tau)$, которая является решением задачи

$$
\left(\frac{\partial}{\partial \tau}+A_{0}\right) K_{0}(x, y ; \tau)=0, \quad K_{0}(x, y ; 0)=\delta(x-y) .
$$

Тогда

$$
G_{0}(x, y)=\int_{0}^{\infty} d \tau K_{0}(x, y ; \tau)
$$

Если рассмотреть оператор Лапласа $A_{1}=A_{0}+V(x)$ с достаточно хорошим потенциалом, то решение задачи

$$
\left(\frac{\partial}{\partial \tau}+A_{1}\right) K_{1}(x, y ; \tau)=0, \quad K_{1}(x, y ; 0)=\delta(x-y)
$$

дает соответствующее фундаментальное решение

$$
G_{1}(x, y)=\int_{0}^{\infty} d \tau K_{1}(x, y ; \tau)
$$


Согласно общей теории для определенного класса потенциалов можно написать формулу для логарифма детерминанта:

$$
\ln \operatorname{det} \frac{A_{1}}{A_{0}}=-\int_{0}^{\infty} \frac{d \tau}{\tau} \operatorname{tr}\left(e^{-A_{1} \tau}-e^{-A_{0} \tau}\right),
$$

где интегральным операторам $e^{-A_{1} \tau}$ и $e^{-A_{0} \tau}$ отвечают $K_{1}(x, y ; \tau)$ и $K_{0}(x, y ; \tau)$ соответственно.

\section{3. КЛАССИЧЕСКОЕ РЕШЕНИЕ}

Пусть даны $N$-мерный вектор $f(x)$ и матрица $B_{\mu}(x)$ размера $N \times N$ с гладкими компонентами. Определим оператор $A_{1}$ следующим образом:

$$
A_{1} f(x)=-D_{x^{\mu}} D_{x_{\mu}} f(x),
$$

где

$$
D_{x_{\mu}} f(x)=\left(\partial_{x_{\mu}}+B_{\mu}(x)\right) f(x) .
$$

Учитывая постановку задачи (4), решение можно искать в виде

$$
K_{1}(x, y ; \tau)=K_{0}(x, y ; \tau) \sum_{n=0}^{\infty} \tau^{n} a_{n}(x, y),
$$

где функция

$$
K_{0}(x, y ; \tau)=\frac{1}{(4 \pi \tau)^{d / 2}} \exp \left(-\frac{|x-y|^{2}}{4 \tau}\right)
$$

является решением задачи (2). Тогда справедливо следующее

ПредлОЖЕНИЕ 1. Функиия (9) является формалъным решением задачи (2), если выполнены соотношения

$$
\begin{gathered}
(x-y)^{\mu} D_{x_{\mu}} a_{0}(x, y)=0, \\
\left((n+1)+(x-y)^{\mu} D_{x_{\mu}}\right) a_{n+1}(x, y)=-A_{1} a_{n}(x, y), \quad n \geqslant 0 .
\end{gathered}
$$

Пусть аргументы $x$ и $y$ принадлежат $\mathbb{R}^{d}$. Оператор $x^{\mu} \partial_{\mu}$ является оператором, считающим степени, поэтому, если предположить, что решение разлагается в ряд Тейлора в окрестности некоторой точки, то на каждый моном вида

$$
x_{1}^{\alpha_{1}} \cdot \ldots \cdot x_{d}^{\alpha_{d}}, \quad \text { где } \quad \alpha_{j} \in \mathbb{N} \cup 0, \quad j=1, \ldots, d,
$$

налагается условие $\alpha_{1}+\cdots+\alpha_{d}=0$. Отсюда немедленно следует альтернатива: либо $\alpha_{j}=0$ для всех $j=1, \ldots, d$, либо найдется номер $j \in 1, \ldots, d$ такой, что $\alpha_{j}<0$. Классическим называется решение, которое разлагается в ряд Тейлора во всех точках рассматриваемой области.

Введем оператор Р-упорядоченной экспоненты для фиксированного поля $-B_{\mu}(x)$ и контура $\gamma$, представляющего собой отрезок, соединяющий начальную и конечную точки. 
ОПРЕДЕЛЕНИЕ 1. Р-экспонента задается формулой

$$
\Phi(x, y):=1+\sum_{n=1}^{\infty}(-1)^{n} \int_{0}^{1} \ldots \int_{0}^{1} d s_{1} \ldots d s_{n} \frac{d z_{1}^{\nu_{1}}}{d s_{1}} \ldots \frac{d z_{n}^{\nu_{n}}}{d s_{n}} B_{\nu_{1}}\left(z_{1}\right) \ldots B_{\nu_{n}}\left(z_{n}\right)
$$

где точки $z_{1}, \ldots, z_{n-1}$ расположены на контуре в порядке нумерации, параметризации $z_{j}(\cdot)$ являются отображениями отрезка $[0,1]$ в часть отрезка от точки $y$ до точки $z_{j+1}$ при $j=2, \ldots, n$ и от точки $y$ до $x$ при $j=1$.

Основные свойства Р-упорядоченной экспоненты таковы:

- имеет место равенство

$$
\Phi(x, x)=1
$$

- функция (12) является решением интегрального уравнения

$$
\Phi(x, y)=1-\int_{0}^{1} d s \frac{d z^{\nu}(s)}{d s} B_{\nu}(z(s)) \Phi(z(s), y)
$$

• обратный оператор имеет вид (см. доказательство в приложении)

$$
\Phi^{-1}(x, y)=\Phi(y, x)
$$

ПреДЛОЖЕНИЕ 2. Функиия $\Phi(x, y)$ является классическим решением в $\mathbb{R}^{d}$ задачи

$$
(x-y)^{\mu} D_{x_{\mu}} a_{0}(x, y)=0,\left.\quad a_{0}(x, y)\right|_{x=y}=1 .
$$

ДокАЗАТЕЛЬСтво. Достаточно заметить, что, выбирая параметризацию в виде $z^{\mu}(s)=(1-s) y^{\mu}+s x^{\mu}$, после интегрирования по частям мы можем представить производную как

$$
\partial_{x_{\mu}}(\Phi(x, y))=-B_{\mu}(x) \Phi(x, y)-\int_{0}^{1} d z^{\nu}(s) s H_{\mu \nu}(z(s), y)
$$

где антисимметричный по индексам оператор $H_{\mu \nu}$ задается формулой

$$
H_{\mu \nu}(z, y):=\partial_{z_{\mu}}\left(B_{\nu}(z) \Phi(x, y)\right)-\partial_{z_{\nu}}\left(B_{\mu}(z) \Phi(x, y)\right) .
$$

ПРЕДЛОЖЕНИЕ 3. Если предположить, что три точки $x, y, z$ лежат на одной прямой, то справедливо равенство

$$
\Phi(x, y)=\Phi(x, z) \Phi(z, y)
$$

ДокАЗАТЕЛЬство. Левая и правая части равенства (19) с учетом предыдущих свойств и условия $(x-y)^{\mu}=$ const $\cdot(x-z)^{\mu}$ коллинеарности векторов удовлетворяют задаче

$$
(x-y)^{\mu}\left(\partial_{x_{\mu}}+B_{\mu}(x)\right) \Psi(x, y)=0,\left.\quad \Psi(x, y)\right|_{x=y}=1,
$$

что доказывает предложение. 
Для решения остальных дифференциальных уравнений в (11) достаточно понять, как устроено решение следующей модельной задачи. Предположим, что задана достаточно гладкая и хорошо убывающая функция $V(x, y)$, разложение которой в ряд Тейлора в окрестности точки $у$ имеет вид

$$
V(x, y)=\sum_{k=0}^{\infty} \frac{(x-y)_{\nu_{1} \ldots \nu_{k}}}{k !} V_{k}^{\nu_{1} \ldots \nu_{k}}(y)
$$

где для удобства введено обозначение $(x-y)_{\nu_{1} \ldots \nu_{n}}:=(x-y)_{\nu_{1}} \cdot \ldots \cdot(x-y)_{\nu_{n}}$. В таком случае справедливо следующее

ПРЕДЛОЖЕНИЕ 4. Классическое решение в $\mathbb{R}^{d}$ уравнения

$$
\left((n+1)+(x-y)^{\mu} D_{x_{\mu}}\right) W_{n+1}(x, y)=V(x, y)
$$

имеет вид

$$
W_{n+1}(x, y)=\int_{0}^{1} d s s^{n} \Phi(x, z(s)) V(z(s), y) .
$$

ДокАзАтЕЛьство. Достаточно сделать замену

$$
W_{n+1}(x, y)=e^{-(n+1) \pi_{\mu} \ln (x-y)^{\mu}} \Phi(x, y) \widetilde{W}_{n+1}(x, y),
$$

где

$$
\pi_{\mu} \ln (x-y)^{\mu}=\sum_{k=1}^{d} \pi_{k} \ln (x-y)^{k}, \quad \pi \in \mathbb{R}^{d}, \quad \sum_{i=1}^{d} \pi_{i}=1 .
$$

Тогда решение записывается в виде

$$
\widetilde{W}_{n+1}(x, y)=\operatorname{const}(y)+\int_{0}^{1} d s s^{-1} e^{(n+1) \pi_{\mu} \ln (z(s)-y)^{\mu}} \Phi^{-1}(z(s), y) V(z(s), y) .
$$

Ответ (22) следует из условия ограниченности решения, предложения 2 и равенства

$$
e^{(n+1) \pi_{\mu} \ln (z(s)-y)^{\mu}}=s^{n+1} e^{(n+1) \pi_{\mu} \ln (x-y)^{\mu}} .
$$

СлЕДСТВИЕ 1. Из предложений 1-3 следует, что рекуррентная система классических решений дифференииальных уравнений (11) с учетом начального условия имеет вид

$$
a_{n+1}(x, y)=-\int_{0}^{1} d s s^{n} \Phi(x, z(s))\left(A_{1} a_{n}(z(s), y)\right) .
$$

\section{4. ФОРМУЛЫ ДИФФЕРЕНЦИРОВАНИЯ Р-ЭКСПОНЕНТЫ}

В построении диаграммной техники ключевую роль играют формулы дифференцирования упорядоченной экспоненты по первому и второму аргументам. В случае ковариантного дифференцирования по первому аргументу подробный вывод приводится в работе [18]. Можно показать, что справедливо 
ПРЕДЛОЖЕНИЕ 5. Имеет место равенство

$$
D_{x_{\mu}} \Phi(x, y)=\int_{0}^{1} d s s \frac{d z^{\nu}}{d s} \Phi(x, z) F_{\nu \mu}(z) \Phi(z, y)
$$

где

$$
F_{\nu \mu}(x)=\partial_{x_{\nu}} B_{\mu}(x)-\partial_{x_{\mu}} B_{\nu}(x)+\left[B_{\nu}(x), B_{\mu}(x)\right],
$$

as - параметр параметризации $z_{\nu}=(1-s) y_{\nu}+s x_{\nu}$.

ДокАЗАтЕльство. Если ввести поле

$$
K_{\mu}(y)=-\int_{0}^{1} d z^{\nu}(s) s H_{\mu \nu}(y, z(s))
$$

и учесть соотношения (17) и (18), то очевидны равенства

$$
D_{x_{\mu}} \Phi(x, y)=K_{\mu}(x), \quad(x-y)^{\nu} K_{\nu}(z) \equiv 0 ;
$$

также можно показать, что

$$
K_{\mu}(x)=-\int_{0}^{1} d s s(x-y)^{\nu}\left(F_{\mu \nu} \Phi(z, y)+B_{\nu}(z) K_{\mu}(z)\right) .
$$

Далее прямой подстановкой соотношения (26) в (29) с учетом свойства (14) можно убедиться в справедливости доказываемой формулы.

Формула дифференцирования упорядоченной экспоненты по второму аргументу выводится при помощи равенства (26). Действительно, если продифференцировать равенство $\Phi^{-1}(x, y) \Phi(x, y)=1$, то с учетом основных свойств (13), (15) и (19) мы получаем

ПРЕДЛОЖЕНИЕ 6. Имеет место равенство

$$
\partial_{x_{\mu}} \Phi(y, x)=\Phi(y, x) B_{\mu}(x)-\int_{0}^{1} d s s \frac{d z^{\nu}}{d s} \Phi(y, z) F_{\nu \mu}(z) \Phi(z, x) .
$$

После замен $x \leftrightarrow y$ и $s \rightarrow 1-s$ формула (30) преобразуется, и справедливо

ПРЕДЛОЖЕНИЕ 7. Имеет место равенство

$$
\partial_{y_{\mu}} \Phi(x, y)=\Phi(x, y) B_{\mu}(y)+\int_{0}^{1} d s(1-s) \frac{d z^{\nu}}{d s} \Phi(x, z) F_{\nu \mu}(z) \Phi(z, y) .
$$

\section{5. ДИАГРАММНАЯ ТЕХНИКА}

5.1. Мотивировка. Следует отметить, что представленная в настоящей работе диаграммная техника не связана с диаграммами Фейнмана. Она используется в основном для компактной записи, удобства проведения вычислений и анализа свойств.

Как уже упоминалось, ключевую роль при построении диаграмматики играют формулы (26), (30) и (31). Действительно, можно заметить, что при ковариантном дифференцировании функция $\Phi(x, y)$ переходит в интеграл от произведения упорядоченных экспонент и напряженности. Следовательно, продолжая дифференцировать, мы будем получать лишь упорядоченные экспоненты и производные от напряженности. Более детальный анализ показывает справедливость этого предположения и позволяет получить способ контроля коэффициентов, возникающих при действии операторами дифференцирования. 
5.2. Основные определения. Диаграммная техника состоит из нескольких основных элементов.

ОПРЕДЕлЕниЕ 2. Функции $\Phi(x, y)$ соответствует линия с аргументами:

$$
x \longrightarrow y \text {. }
$$

ОПРЕДЕЛЕНИЕ 3. Функции между упорядоченными экспонентами сопоставляется окружность, зависящая от следующих параметров:

1) набор греческих индексов $\mu_{1}, \ldots, \mu_{n}$, которому отвечает

$$
\nabla_{\mu_{1}} \ldots \nabla_{\mu_{n-1}}\left(d(z-y)^{\rho} F_{\rho \mu_{n}}(z)\right)
$$

где все операторы действуют на переменную $z$ и $\nabla_{x_{\mu}}(\bullet)=\partial_{x_{\mu}}(\bullet)+\left[B_{\mu}(x), \cdot\right]$;

2) параметр параметризации $s^{k}$ в соответствующей степени;

3) параметр $y \rightarrow x$, который символизирует интеграл по прямой от точки $y$ до точки $x$ с параметризацией $z_{\nu}=(1-s) y_{\nu}+s x_{\nu}$.

Например, формула (26) переписывается в виде

$$
\int_{y}^{x} d z^{\nu} s \Phi(x, z) F_{\nu \mu}(z) \Phi(z, y)=x \longrightarrow s^{1}(y \rightarrow x)
$$

где две линии отвечают функциям $\Phi(x, z)$ и $\Phi(z, y)$, окружность с параметрами $\mu$, $(y \rightarrow x)$ и $s^{1}$ отвечает интегрированию от $x$ до $y$ формы $d z_{\nu}(s) F_{\nu \mu}(z(s))$ с весом $s$. При интегрировании окружность проходит указанный отрезок.

ОПРЕДЕЛЕНИЕ 4. Пусть $z_{\nu}=(1-s) y_{\nu}+s x_{\nu}$, тогда конструкции вида

$$
\int_{0}^{1} d s s^{n} x \longrightarrow z(s) \cdot z(s) \longrightarrow \text { (любая диаграмма) }
$$

соответствует диаграмма

$$
x \stackrel{s^{n+1}(y \rightarrow x)}{X} \text { (любая диаграмма). }
$$

Основой для введения последнего определения послужила формула (25).

5.3. Основной пример. Чтобы понять, как устроена диаграммная техника, имеет смысл рассчитать первую итерацию системы (25). В нулевом порядке решение системы (11) диктуется формулой $a_{0}(x, y)=\Phi(x, y)$ из определения 1 (см. формулу (12)). Далее применяются операторы Лапласа и интегрирования.

Во-первых, согласно формуле (26)

$$
D_{x_{\mu}} \Phi(x, y)=\int_{0}^{1} d s_{1} s_{1} \frac{d z^{\nu}}{d s_{1}} \Phi(x, z) F_{\nu \mu}(z) \Phi(z, y)
$$

или

$$
D_{x_{\mu}} x \longrightarrow y=x \stackrel{\mu s_{1}^{1}(y \rightarrow x)}{-} y
$$


Во-вторых, из соотношений (26) и (31) следует, что вторая ковариантная производная имеет вид

$$
\begin{aligned}
D_{x^{\mu}} D_{x_{\mu}} \Phi(x, y) & =\int_{y}^{x} d z^{\nu}\left(s_{1}\right) s_{1} \int_{z}^{x} d z^{\prime \rho}\left(s_{2}\right) s_{2} \Phi\left(x, z^{\prime}\right) F_{\rho \mu}\left(z^{\prime}\right) \Phi\left(z^{\prime}, z\right) F_{\nu \mu}(z) \Phi(z, y)+ \\
& +\int_{y}^{x} d z^{\nu}\left(s_{1}\right) s_{1}^{2} \int_{z}^{x} d z^{\prime \rho}\left(1-s_{2}\right) \Phi\left(x, z^{\prime}\right) F_{\rho \mu}\left(z^{\prime}\right) \Phi\left(z^{\prime}, z\right) F_{\nu \mu}(z) \Phi(z, y)+ \\
& +\int_{y}^{x} d z^{\nu}\left(s_{1}\right) s_{1}^{2} \Phi(x, z) F_{\nu \mu}(z) \int_{y}^{z} d z^{\prime \rho}\left(s_{2}\right) s_{2} \Phi\left(z, z^{\prime}\right) F_{\rho \mu}\left(z^{\prime}\right) \Phi\left(z^{\prime}, y\right)+ \\
& +\int_{y}^{x} s_{1} \Phi(x, z)\left(s_{1} \partial_{z^{\mu}}\left(d z^{\nu}\left(s_{1}\right) F_{\nu \mu}(z)\right)\right) \Phi(z, y)+ \\
& +\int_{y}^{x} d z^{\nu}\left(s_{1}\right) s_{1}^{2} \Phi(x, z) B^{\mu}(z) F_{\nu \mu}(z) \Phi(z, y)- \\
& -\int_{y}^{x} d z^{\nu}\left(s_{1}\right) s_{1}^{2} \Phi(x, z) F_{\nu \mu}(z) B^{\mu}(z) \Phi(z, y)
\end{aligned}
$$

что на языке диаграмм эквивалентно

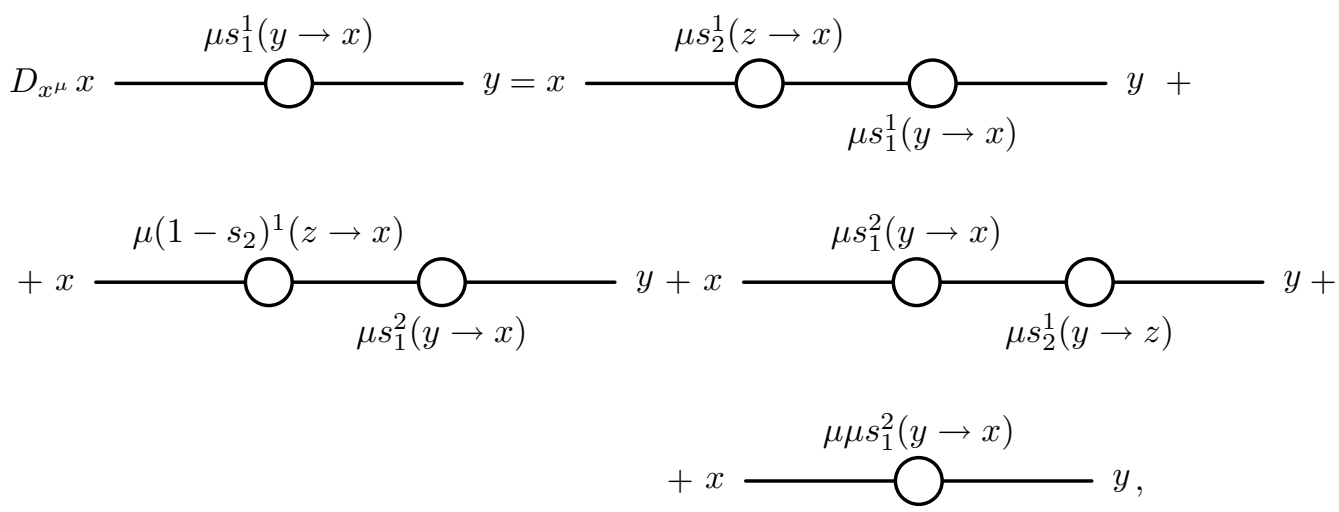

где первые три слагаемых в (33) соответствуют первым трем диаграммам, а последние три образуют действие оператора $\nabla_{\mu}$ на напряженность и изображаются четвертой диаграммой.

В-третьих, после интегрирования мы имеем

$$
\begin{aligned}
& \left.\int_{y}^{x} d s \Phi\left(x, z^{\prime}(s)\right)\left(D_{x^{\mu}} D_{x_{\mu}} x \longrightarrow y\right)\right|_{x=z^{\prime}(s)}= \\
& =x \longrightarrow s_{s^{1}(y \rightarrow x)}^{\mu s_{2}^{1}\left(z \rightarrow z^{\prime}\right)}\left(\bigcirc_{\mu s_{1}^{1}\left(y \rightarrow z^{\prime}\right)}^{O} y+\right.
\end{aligned}
$$

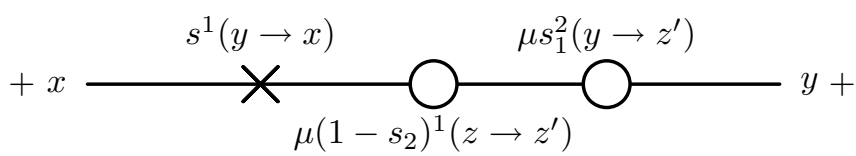




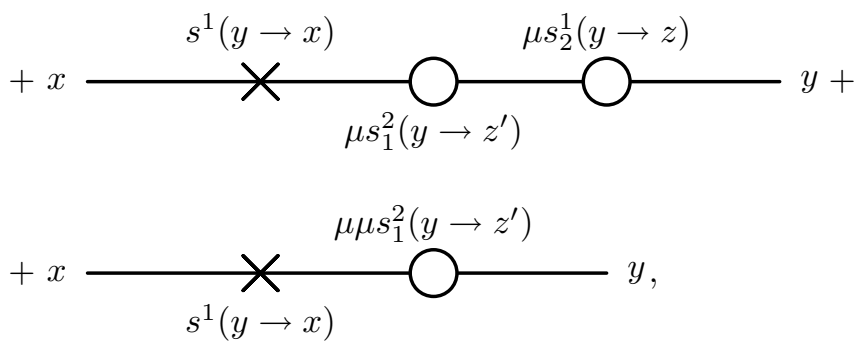

где используется стандартная параметризация $z_{\mu}^{\prime}(s)=(1-s) y_{\mu}+s x_{\mu}$ и определение 4.

5.4. Теорема о дифференцировании диаграмм. Основная задача данного пункта - научиться применять ковариантную производную к произвольной диаграмме, а также упростить диаграммную технику и отказаться от лишних параметров.

ПрЕДЛОЖЕНИЕ 8. Пусть заданы две непрерывные функиии $f(x)$ u $g(x)$ и параметризачии

$$
z_{\mu}=(1-s) y_{\mu}+s y_{\mu}^{\prime}, \quad x_{\mu}=(1-t) z_{\mu}+t y_{\mu}^{\prime} .
$$

Тогда имеет место равенство

$$
\int_{y}^{y^{\prime}} d z_{\rho} \int_{z}^{y^{\prime}} d x_{\nu} s^{n} t^{p} f(z) g(x)=\int_{y}^{y^{\prime}} d x_{\nu}^{\prime} \int_{y}^{x^{\prime}} d z_{\rho}^{\prime n}\left(s^{\prime}\right)\left(t^{\prime}\right)^{n+p}\left(\frac{1-s^{\prime}}{1-s^{\prime} t^{\prime}}\right)^{p} f\left(z^{\prime}\right) g\left(x^{\prime}\right),
$$

где

$$
x_{\mu}^{\prime}=\left(1-t^{\prime}\right) y_{\mu}+t^{\prime} y_{\mu}^{\prime}, \quad z_{\mu}^{\prime}=\left(1-s^{\prime}\right) y_{\mu}+s^{\prime} x_{\mu}^{\prime} .
$$

ДокАзАтЕЛьство. Достаточно сделать замену

$$
t \rightarrow t^{\prime}=(1-s) t+s, \quad\left(s, t^{\prime}\right) \in[0,1] \times[s, 1] \rightarrow\left(s, t^{\prime}\right) \in\left[0, t^{\prime}\right] \times[0,1],
$$

а затем воспользоваться теоремой Фубини и сделать еще одну замену $s \rightarrow s / t^{\prime}$.

ПРЕДЛОЖЕНИЕ 9. Имеет место соотношение

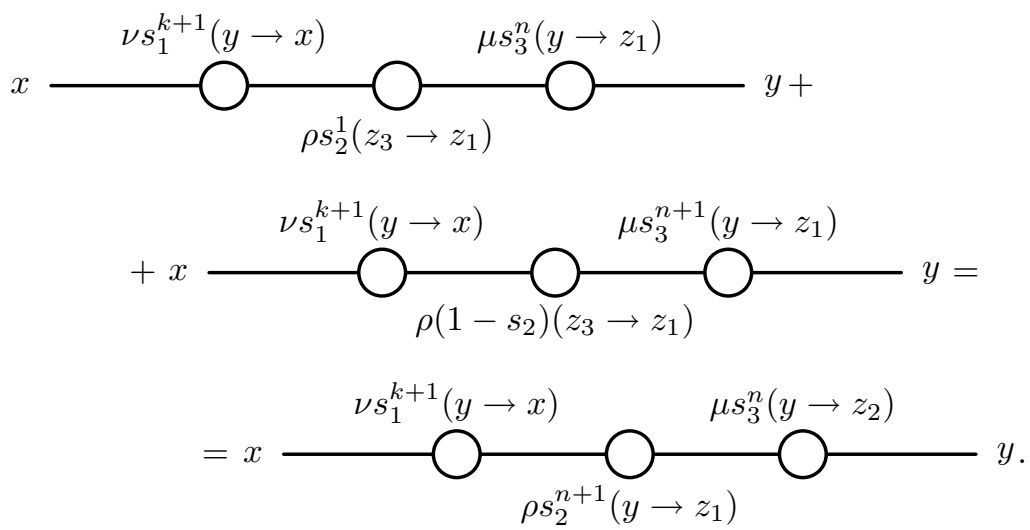


ДокАЗАТЕЛЬСтво может быть достигнуто в несколько этапов. Из предложения 8 о перестановке интегралов следует, что

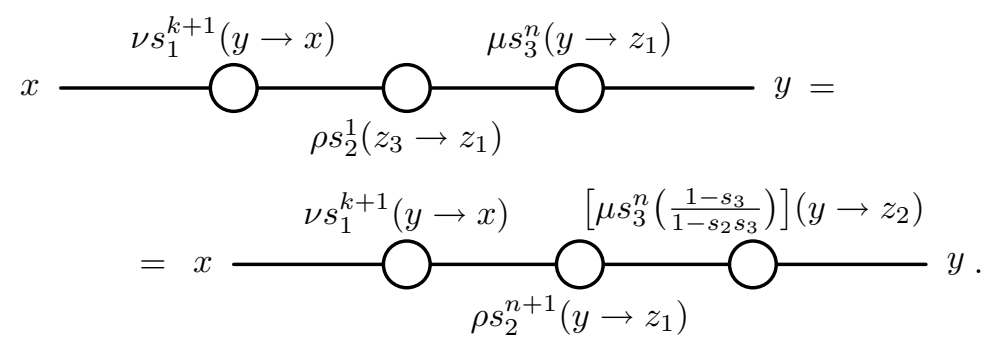

Аналогично из предложения 8 следует результат для второй диаграммы:

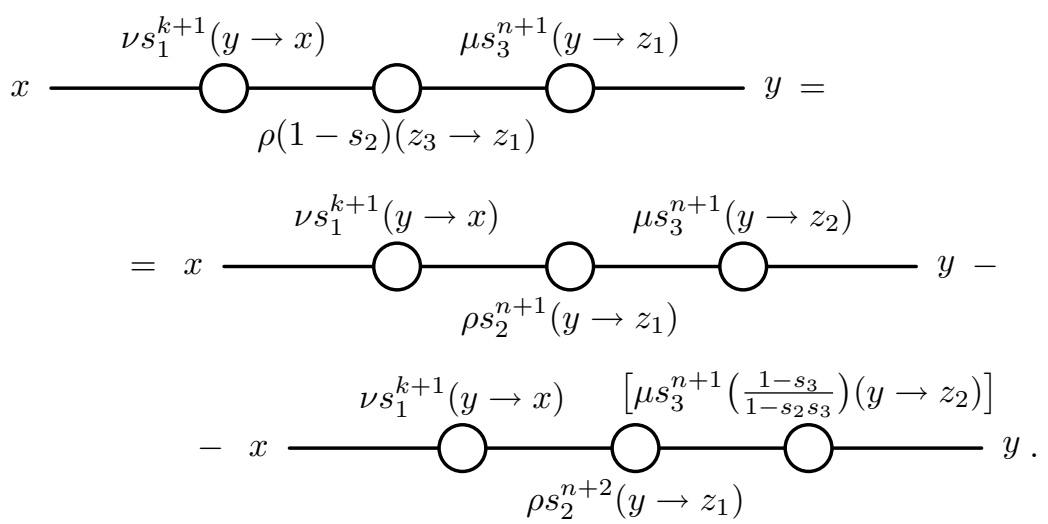

При этом выполняется следующее соотношение для параметров:

$$
s_{2}^{n+1} s_{3}^{n}\left(\frac{1-s_{3}}{1-s_{2} s_{3}}\right)+s_{2}^{n+1} s_{3}^{n+1}-s_{2}^{n+2} s_{3}^{n+1}\left(\frac{1-s_{3}}{1-s_{2} s_{3}}\right)=s_{2}^{n+1} s_{3}^{n} .
$$

ПРЕДЛОЖЕНИЕ 10. Имеет место соотношение

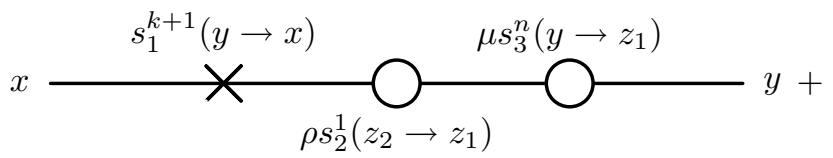

$$
\begin{aligned}
& +x \frac{s_{1}^{k+1}(y \rightarrow x)}{\times} \overbrace{\rho\left(1-s_{2}\right)\left(z_{2} \rightarrow z_{1}\right)}^{\mu s_{3}^{n+1}\left(y \rightarrow z_{1}\right)} \overbrace{}^{(y-1}= \\
& =x \longrightarrow \frac{s_{1}^{k+1}(y \rightarrow x)}{\times} \bigodot_{\rho s_{2}^{n+1}}{ }_{\left(y \rightarrow z_{1}\right)}^{\mu s_{3}^{n}\left(y \rightarrow z_{2}\right)}
\end{aligned}
$$


ПрЕДЛОЖЕНИЕ 11. Имеет место соотношение

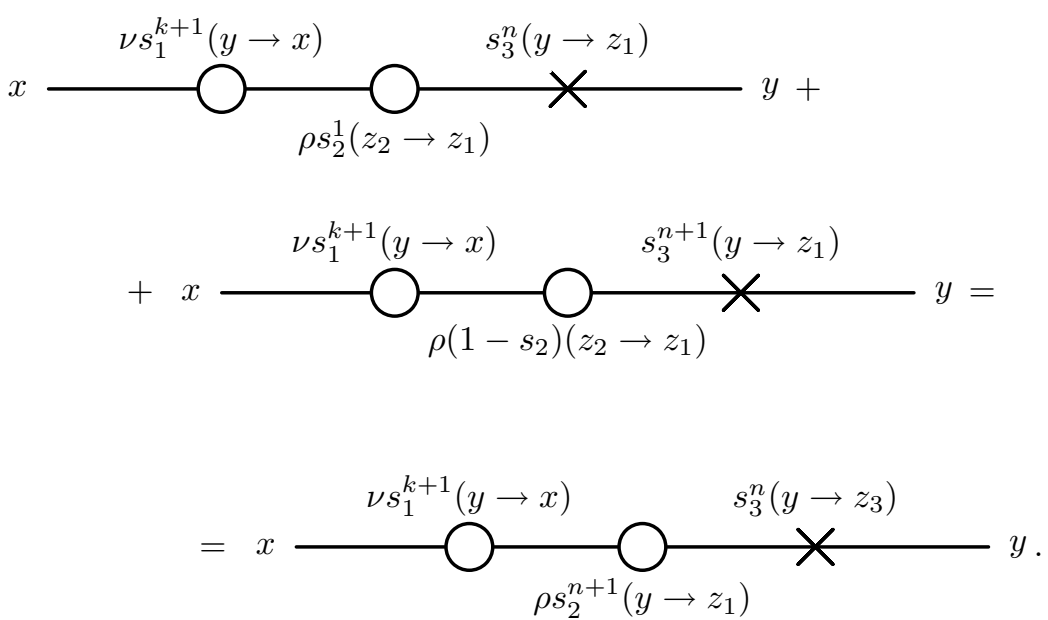

СлеДСТвИЕ 2. Из предложений 9-11 следует, что можно отказатъся от третъего параметра в определении 3 , поскольку интегрирование всегда проходит от точки у до ближайщей точки, лежащей слева.

СлеДСТвиЕ 3. Формула для первого порядка записъвается как

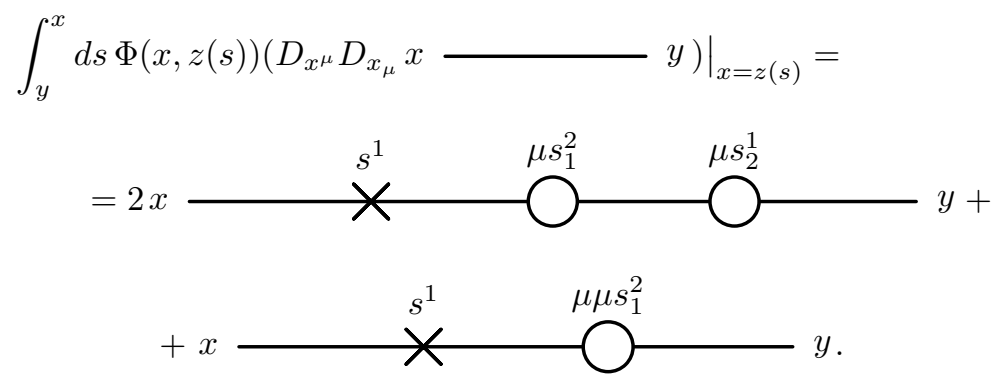

Подводя итог, сформулируем теорему о дифференцировании диаграммы.

Теорема 1. Пусть имеется произвольная диаграмма с $i$ линиями, $k$ окружностями и $ј$ крестиками и к ней применяется операция $D_{\rho} ;$ пусть $t$ - новая параметризачия. Тогда возможны три случая.

1. Когда производная действует на линию, последняя заменяется на окружность с двумя линиями по бокам и параметрами $\rho$ и в степени на единицу больше, чем степень второго параметра справа стоящей вершины (если же линия самая правая, то степень равна 1). При этом степень каждого веса, стоящего слева от дибберенцируемой линии, увеличивается на единицу. В результате мъ имеем $і$ диаграмм.

2. Когда производная действует на окружность, то степенъ ее второго параметра увеличивается на единичу, а индекс $\rho$ прикрепляется слева к остальным. При этом степень каждого второго параметра вершин, стоящих слева, увеличивается на единицу. В результате мы имеем $k$ диаграмм. 
3. Когда производная действует на крестик, то диаграмма обращается в нуль. Итак, после дифферениирования мы имеем $i+k$ диаграмм.

Например,

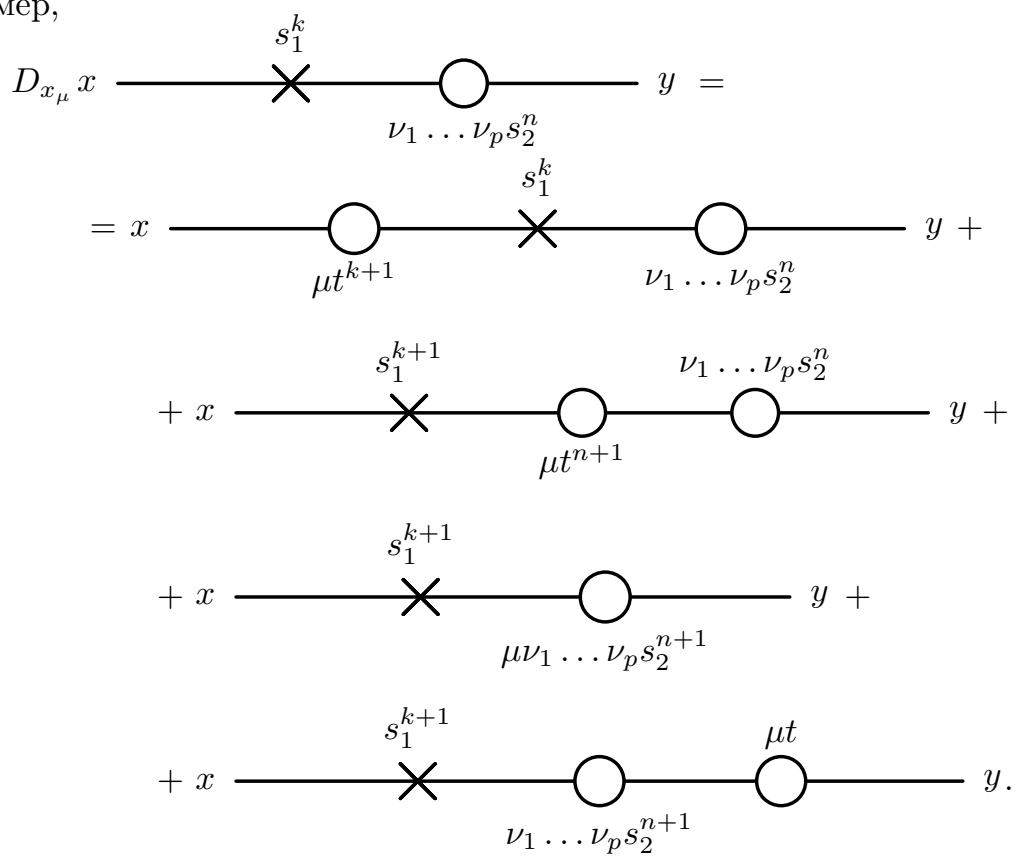

СледСтвиЕ 4. Операция дифферениирования распадается на операцию $\nabla$, действующую на каждую окружность, и на операцию добавления новой окружности ( с соблюдением правила повышения степеней параметров параметризации).

5.5. Первый порядок. Первый порядок записывается в виде

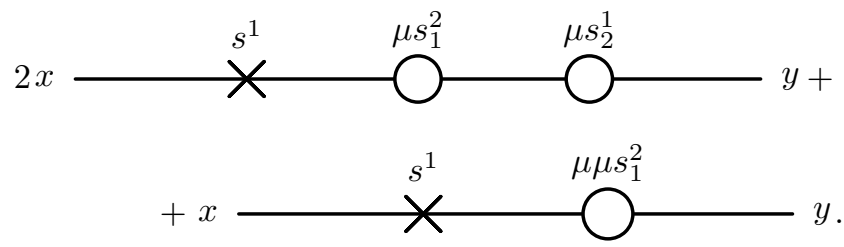

Чтобы узнать вклад при подсчете следа, следует взять $x=y$. Таким образом, требуется узнать нулевой член разложения в ряд Тейлора. Легко видеть, что первое слагаемое диаграммы начинается с квадратичного члена, поскольку каждая окружность с одним индексом содержит линейный вклад. В свою очередь второе слагаемое дает результат $\left.\nabla^{\mu}\left((x-y)^{\nu} F_{\nu \mu}\right)\right|_{x=y}$, который, очевидно, равен нулю ввиду антисимметричности напряженности.

5.6. Второй порядок. Для подсчета второго порядка достаточно применить дважды ковариантную производную к первому порядку и взять интеграл. Далее нужно положить $x=y$ и выбрать вклады с ненулевыми следами. Необходимо 
учесть, что, во-первых, каждая окружность должна содержать не менее двух греческих индексов, иначе вклад при $x=y$ будет нулевым; во-вторых, окружность с двумя одинаковыми греческими индексами дает нулевой вклад; в-третьих, диаграмма с одной окружностью, которая содержит более двух греческих индексов, дает нулевой вклад. С учетом этих замечаний формула принимает вид

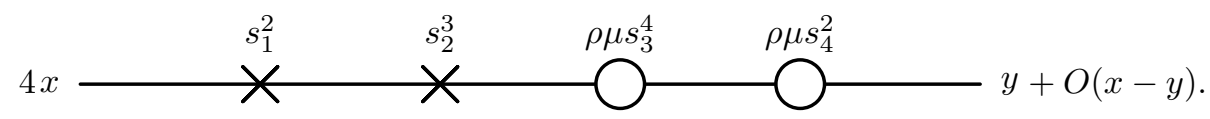

При $x=y$ все упорядоченные экспоненты перейдут в единицы, поэтому, используя равенство

$$
\left.\left(\nabla_{\rho}\left((x-y)^{\nu} F_{\nu \mu}(x)\right)\right)\right|_{x=y}=F_{\rho \mu}(x)
$$

мы можем записать ответ как

$$
\frac{1}{12} F_{\rho \mu}(x) F^{\rho \mu}(x)
$$

\section{6. ОПЕРАТОРЫ, ДЕЙСТВУЮЩИЕ НА МАТРИЦЫ}

6.1. Мотивировка. Правила диаграммной техники, описанные в предыдущем разделе, позволяют достаточно легко находить коэффициенты. В качестве примера мы получили стандартные результаты (35) для первого и второго порядков. Однако процедура является рекуррентной. В данном разделе приводится формализм, позволяющий вывести окончательный ряд для теплового ядра ковариантного оператора Лапласа при $x=y$.

6.2. Матрицы. Ранее было показано, что вершины в диаграммах содержат два параметра, поэтому можно каждой диаграмме сопоставить матрицу, которая будет иметь две строки. Количество столбцов в такой матрице равно суммарному количеству окружностей и крестиков в диаграмме.

Основные свойства матриц таковы:

1) матрицы содержат две строки и $n$ столбцов с сохранением очередности, где $n-$ суммарное количество вершин в диаграмме;

2) в первой строке элементы могут быть двух видов: это либо единица, если столбец отвечает крестику на диаграмме, либо набор греческих индексов, если столбец отвечает окружности;

3) вторая строка содержит степени вторых параметров вершин;

4) единицей $\mathbb{1}$ обозначается элемент, который не содержит столбцов.

Таким образом, получаются матрицы вида $\mathbb{1}, \mathrm{M}^{2 \times 1}, \mathrm{M}^{2 \times 2}, \ldots$. Следует отметить, что в каждом порядке теплового ядра присутствует конечное число матриц конечного размера. Сложение таких матриц возможно, только если они имеют одинаковые элементы и одинаковый размер. Ясно, что базис в этом случае не более чем счетный, поскольку и первая, и вторая строки могут содержать элементы лишь из счетного набора. 
6.3. Определения операторов. В соответствии с перечисленными выше правилами любую диаграмму можно записать в виде матрицы, поэтому удобно ввести оператор, вычисляющий диаграмму в точке $x=y$.

ОПРЕДЕЛЕНИЕ 5. Оператор $\Upsilon$ сопоставляет каждому набору греческих индексов соответствующую по определению 3 конструкцию, в которой произведена замена дифференциала $d(z-y)$ на $z-y$ и выбрана точка $z=y=x$, а затем произведение элементов верхней строки делится на произведение элементов нижней строки.

Например,

$$
\Upsilon \mathbb{1}=1, \quad \Upsilon\left(\begin{array}{cc}
1 & \nu \mu \\
2 & 3
\end{array}\right)=\left.\frac{1}{6} \nabla_{z^{\nu}}\left((z-y)^{\rho} F_{\rho \mu}(z)\right)\right|_{z=y=x} .
$$

Ранее было отмечено, что ковариантная производная от диаграммы сводится либо к оператору добавления окружности с индексом, либо к оператору добавления индекса (с соблюдением правила изменения степеней весов). Также оператор интегрирования сводится к оператору добавления крестика. Легко заметить, что на матрицах можно ввести следующие аналогичные операторы.

- Оператор домножения:

$$
\begin{aligned}
B^{\mu}\left(\begin{array}{llll}
\nu_{1} & \nu_{2} & \ldots & \nu_{n} \\
k_{1} & k_{2} & \ldots & k_{n}
\end{array}\right)=\left(\begin{array}{cccc}
\mu \nu_{1} & \nu_{2} & \ldots & \nu_{n} \\
k_{1}+1 & k_{2} & \ldots & k_{n}
\end{array}\right)+\cdots+ \\
\\
\\
+
\end{aligned}
$$

- Оператор добавления столбца:

$$
\begin{aligned}
A^{\mu} & \left(\begin{array}{llll}
\nu_{1} & \nu_{2} & \ldots & \nu_{n} \\
k_{1} & k_{2} & \ldots & k_{n}
\end{array}\right)=\left(\begin{array}{ccccc}
\mu & \nu_{1} & \nu_{2} & \ldots & \nu_{n} \\
k_{1}+1 & k_{1} & k_{2} & \ldots & k_{n}
\end{array}\right)+\cdots+ \\
& +\left(\begin{array}{ccccccccc}
\nu_{1} & \nu_{2} & \ldots & \mu & \nu_{n} \\
k_{1}+1 & k_{2}+1 & \ldots & k_{n}+1 & k_{n}
\end{array}\right)+\left(\begin{array}{cccccc}
\nu_{1} & \nu_{2} & \ldots & \nu_{n} & \mu \\
k_{1}+1 & k_{2}+1 & \ldots & k_{n}+1 & 1
\end{array}\right) .
\end{aligned}
$$

- Оператор интегрирования:

$$
S_{l}^{\mu}\left(\begin{array}{llll}
\nu_{1} & \nu_{2} & \ldots & \nu_{n} \\
k_{1} & k_{2} & \ldots & k_{n}
\end{array}\right)=\left(\begin{array}{ccccc}
\mu & \nu_{1} & \nu_{2} & \ldots & \nu_{n} \\
l & k_{1} & k_{2} & \ldots & k_{n}
\end{array}\right)
$$

Используя эти определения, можно записать тепловое ядро (9) для ковариантного оператора Лапласа в виде

$$
K_{1}(x, x ; \tau)=\Upsilon\left(1+\sum_{n=1}^{\infty} \tau^{n} \prod_{k=1}^{n} S_{k}^{1}\left(A^{\mu_{k}}+B^{\mu_{k}}\right)\left(A^{\mu_{k}}+B^{\mu_{k}}\right)\right) \mathbb{1}
$$

6.4. Коммутаторы операторов. Основная идея состоит в том, чтобы воспользоваться коммутаторами определенных выше операторов и передвинуть в (40) операторы домножения и добавления столбца направо. В этом случае удастся избавиться от рекуррентной процедуры. Легко проверить, что справедливо 
ПРЕДЛОЖЕНИЕ 12. Имеют место равенства

$$
\begin{array}{ll}
A^{\mu} S_{k}^{1}=S_{k+1}^{1} A^{\mu}+S_{k+1}^{\mu} S_{k}^{1}, & A^{\mu} S_{k}^{\nu_{1} \ldots \nu_{n}}=S_{k+1}^{\nu_{1} \ldots \nu_{n}} A^{\mu}+S_{l+1}^{\mu \nu_{1} \ldots \nu_{n}} S_{k}^{1}, \\
B^{\mu} S_{k}^{1}=S_{k+1}^{1} B^{\mu}, & B^{\mu} S_{k}^{\nu_{1} \ldots \nu_{n}}=S_{k+1}^{\nu_{1} \ldots \nu_{n}} B^{\mu}+S_{k+1}^{\mu \nu_{1} \ldots \nu_{n}} S_{k}^{1} .
\end{array}
$$

В этом предложении приводятся только необходимые для дальнейших доказательств соотношения.

6.5. Свойства операторов интегрирования. Из соотношений (41) видно, что коммутаторы операторов $A$ и $B$ с $S$-операторами вновь переходят в $S$-операторы. Следовательно, всю конструкцию для теплового ядра можно свести к сумме комбинаций операторов интегрирования. В силу этого необходимо определить несколько конструкций, которые облегчат дальнейшее описание.

Прежде всего следует ввести следующий операторный ряд. Пусть $I_{n}=\{1, \ldots, n\}$ и мультииндекс $\mu_{I_{n}}=\mu_{1} \ldots \mu_{n}$, тогда

$$
\Sigma_{l}^{\mu_{I_{n}}}=\sum_{\sigma} S_{l+\sharp \cup_{i=1}^{k} \sigma_{i}}^{\mu_{\sigma_{k}}} \ldots S_{l+\sharp \sigma_{1} \cup \sigma_{2}}^{\mu_{\sigma_{2}}} S_{l+\sharp \sigma_{1}}^{\mu_{\sigma_{1}}},
$$

где суммирование ведется по разбиениям, удовлетворяющим следующим свойствам:

- $\sigma_{j} \in I_{n}$ для всех $j \in\{1, \ldots, k\}$,

- $\sigma_{i} \cap \sigma_{j}=\varnothing$ при $i \neq j$,

- $\cup_{i=1}^{k} \sigma_{i}=I_{n}$ и $\sigma_{s}(i)>\sigma_{s}(j)$ при любых $s \in\{1, \ldots, k\}$ и любых $i, j \in\left\{1, \ldots, \sharp \sigma_{s}\right\}$ таких, что $i>j$.

Здесь оператор $\sharp$ считает количество элементов в множестве. Сумма в формуле (42) является конечной, поэтому никаких проблем, связанных со сходимостью, не возникает.

Если подействовать таким оператором на $\mathbb{1}$ (это не налагает никаких ограничений, поскольку любую матрицу можно получить из единицы при помощи некоторого набора операторов интегрирования), а затем применить оператор $\Upsilon$, то в итоге получится некоторая сумма, содержащая напряженности и числовые коэффициенты. Для того чтобы предъявить точный результат, следует обратить внимание на несколько утверждений.

ПРЕДЛОЖЕНИЕ 13. Имеют место равенства

$$
\begin{aligned}
\nabla_{\mu_{I_{n}}}\left((x-y)^{\rho} F_{\rho \nu}(x)\right) & =(x-y)^{\rho} \nabla_{\mu_{I_{n}}} F_{\rho \nu}(x)+\sum_{k=1}^{n} \nabla_{\mu_{I_{n} \backslash k}} F_{\mu_{k} \nu}(x), \\
\left.\nabla_{\mu_{I_{n}}}\left((x-y)^{\rho} F_{\rho \nu}(x)\right)\right|_{x=y} & =\sum_{k=1}^{n} \nabla_{\mu_{I_{n} \backslash k}} F_{\mu_{k} \nu}(x) .
\end{aligned}
$$

ПРЕДЛОЖЕНИЕ 14. Имеет место равенство

$$
\widehat{S}_{l}^{\mu_{I_{n} \nu}}:=\Upsilon S_{l}^{\mu_{I_{n}} \nu} \mathbb{1}=\frac{1}{l} \sum_{k=1}^{n} \nabla_{\mu_{I_{n} \backslash k}} F_{\mu_{k} \nu}(x) ;
$$

здесъ для удобства мъь ввели дополнительное обозначение $\widehat{S}_{l}^{\mu_{I_{n}} \nu}$. 
ПреДЛОЖЕНИЕ 15. Для любых наборов индексов $I$ u $J$ u натуральных $l$ u $k$ справедливо соотношение

$$
\begin{aligned}
\Upsilon\left(S_{l}^{\mu_{I} \nu} S_{k}^{\mu_{J} \rho} \mathbb{1}\right) & =\left(\Upsilon S_{l}^{\mu_{I} \nu} \mathbb{1}\right)\left(\Upsilon S_{l}^{\mu_{J} \rho} \mathbb{1}\right)= \\
& =\frac{1}{l \cdot k}\left(\sum_{i=1}^{\sharp I} \nabla_{\mu_{I \backslash i}} F_{\mu_{i} \nu}(x)\right)\left(\sum_{j=1}^{\sharp J} \nabla_{\mu_{J \backslash j}} F_{\mu_{j} \nu}(x)\right)=\widehat{S}_{l}^{\mu_{I} \nu} \widehat{S}_{k}^{\mu_{J} \rho} .
\end{aligned}
$$

Доказательство последнего утверждения вытекает из предложений 13 и 14. Ясно, что данное свойство распространяется на любое количество операторов интегрирования. В итоге можно утверждать, что конструкция $\Upsilon \Sigma \mathbb{1}$ полностью известна и может быть выражена через напряженность и ее производные в соответствии со следующим утверждением.

ПрЕДЛОЖЕНИЕ 16. Имеет место равенство

$$
\Upsilon \Sigma_{l}^{\mu_{I_{n}}} \mathbb{1}=\sum_{\sigma} \widehat{S}_{l+\sharp \cup_{i=1}^{k} \sigma_{i}}^{\mu_{\sigma_{k}}} \ldots \widehat{S}_{l+\sharp \sigma_{1} \cup \sigma_{2}}^{\mu_{\sigma_{2}}} \widehat{S}_{l+\sharp \sigma_{1}}^{\mu_{\sigma_{1}}},
$$

где суммирование ведется по правилам, описанным выше.

ЗАмЕчАниЕ 1. Предложение 15 справедливо и в случае замены операторов интегрирования $S$ на операторы $\Sigma$. Его также можно распространить на произвольное количество множителей.

6.6. Ряд для теплового ядра. Формула (40) представляет собой ряд Тейлора по степеням собственного времени. Отдельный коэффициент Сили-Девитта имеет вид

$$
a_{n}(x, x)=\Upsilon\left(\prod_{k=1}^{n} S_{k}^{1}\left(A^{\mu_{k}}+B^{\mu_{k}}\right)\left(A^{\mu_{k}}+B^{\mu_{k}}\right)\right) \mathbb{1} .
$$

Для того чтобы записать коэффициент в терминах напряженности и ее производных, нам потребуется следующее

ПрЕДЛОЖЕНИЕ 17. Пусть $I$ - набор индексов $c \sharp I=n$, a l- некоторое натуральное число. Тогда имеет место операторное равенство

$$
(A+B)^{\mu_{I}} S_{l}^{1}=\sum_{k=0}^{n} \sum_{\sigma_{k}} \Sigma_{l+k}^{\mu_{I \backslash \sigma_{k}}} S_{l+k}^{1}(A+B)^{\mu_{\sigma_{k}}},
$$

где суммирование ведется по всем подмножествам $\sigma_{k} \in I$, удовлетворяющим условиям $\sharp \sigma_{k}=k u \sigma_{k}(i)>\sigma_{k}(j)$ для всех $i, j \in\{1, \ldots, k\}$ maких, что $i>j$.

ДокАЗАТЕльство. Для проверки формулы (48) можно воспользоваться математической индукцией. Для $n=1$ все очевидно:

$$
(A+B)^{\mu_{1}} S_{l}^{1}=S_{l+1}^{1}(A+B)^{\mu_{1}}+S_{l+1}^{\mu_{1}} S_{l}^{1} .
$$

Предположим, что равенство (48) выполняется для $n=k$, докажем его для $n=k+1$ :

$$
\begin{aligned}
(A+B)^{\rho}(A+B)^{\mu_{I}} S_{l}^{1} & =\sum_{k=0}^{n-1} \sum_{\sigma_{k}}(A+B)^{\rho} \Sigma_{l+k}^{\mu_{I \backslash \sigma_{k}}} S_{l+k}^{1}(A+B)^{\mu_{\sigma_{k}}}= \\
& =\sum_{k=0}^{n} \sum_{\sigma_{k}}\left(\Sigma_{l+k}^{\rho \mu_{I \backslash \sigma_{k}}} S_{l+k}^{1}(A+B)^{\mu_{\sigma_{k}}}+\Sigma_{l+k+1}^{\mu_{I \sigma_{k}}} S_{l+k+1}^{1}(A+B)^{\rho \mu_{\sigma_{k}}}\right) .
\end{aligned}
$$

Осталось переобозначить индексы, откуда и следует (48). Предложение доказано. 
Подстановку формулы (48) в (47) лучше делать в несколько шагов.

ПРЕДЛОЖЕНИЕ 18. Пусть $I=I_{2 n}$, тогда

$$
\begin{aligned}
& \prod_{k=1}^{n} S_{k}^{1}\left(A^{\mu_{2 k}}+B^{\mu_{2 k}}\right)\left(A^{\mu_{2 k-1}}+B^{\mu_{2 k-1}}\right)= \\
& =\sum_{p_{1}=0}^{2} \sum_{p_{2}=0}^{4-p_{1}} \cdots \sum_{p_{n-1}=0}^{2(n-1)-\sum_{k=1}^{n-2} p_{k}} \sum_{\sigma} S_{n}^{1} \Sigma_{n+1-p_{1}}^{\mu_{\sigma_{p_{1}}}} S_{n+1-p_{1}}^{1} \Sigma_{n+2-p_{2}}^{\mu_{\sigma_{p_{2}}}} S_{n+2-p_{2}}^{1} \times \cdots \\
& \cdots \times \Sigma_{2 n-1-p_{n-1}}^{\mu_{\sigma_{p_{n-1}}}} S_{2 n-1-p_{n-1}}^{1}(A+B)^{\mu_{I \backslash \cup_{s=1}^{n-1} \sigma_{p_{s}}},}
\end{aligned}
$$

где суммирование ведется по всем возможным подмножествам $\sigma_{p_{k}}$, удовлетворяющим условиям $\sharp \sigma_{p_{k}}=p_{k}, \sigma_{p_{k}} \subset\left(I_{2 n} \backslash I_{2(n-k)}\right) \backslash\left(\cup_{j=1}^{k-1} \sigma_{p_{j}}\right)$ u $\sigma_{p_{k}}(i)>\sigma_{p_{k}}(j)$ для всех $i, j \in\{1, \ldots, k\}$ таких, что $i>j$.

Для доказательства равенства (50) нужно несколько раз применить формулу (48).

ПреДЛОЖЕНИЕ 19. Пусть I - некоторый набор индексов, тогда

$$
\Upsilon(A+B)^{\mu_{I}} \mathbb{1}=\Upsilon \Sigma_{0}^{\mu_{I}} \mathbb{1}=\widehat{\Sigma}_{0}^{\mu_{I}} .
$$

Это утверждение следует из определения операторов. На последнем этапе нужно подействовать оператором (50) на единицу и затем умножить справа на оператор $\Upsilon$.

С учетом полученных результатов сформулируем окончательную теорему.

Теорема 2. Пусть $\mu_{2 k-1}=\mu_{2 k}$ для всех $k \in\{1, \ldots, n\}$, тогда

$$
\begin{aligned}
& a_{n}(x, x)=\sum_{p_{1}=0}^{2} \sum_{p_{2}=0}^{4-p_{1}} \cdots \sum_{p_{n-1}=0}^{2(n-1)-\sum_{k=1}^{n-2} p_{k}} \sum_{\sigma} \frac{\widehat{\Sigma}_{n+1-p_{1}}^{\mu_{\sigma_{p_{1}}}}}{n+1-p_{1}} \frac{\widehat{\Sigma}_{n+2-p_{2}}^{\mu_{\sigma_{p_{2}}}}}{n+2-p_{2}} \times \cdots \\
& \cdots \times \frac{\widehat{\Sigma}_{2 n-1-p_{n-1}}^{\mu_{\sigma_{p_{n-1}}}}}{2 n-1-p_{n-1}} \frac{\widehat{\Sigma}_{0}^{\mu_{I \backslash \cup_{s=1}^{n-1} \sigma_{p_{s}}}}}{n},
\end{aligned}
$$

где суммирование по б подчиняется правилам, описанным в предложении 18.

6.7. Третий порядок. В качестве примера рассчитаем результаты в третьем порядке, которые впервые были получены в работе [8]. Для этого следует воспользоваться формулами (44), (46) и (52), адаптированными для конкретного случая:

$$
a_{3}(x, x)=\sum_{p_{1}=0}^{2} \sum_{p_{2}=0}^{4-p_{1}} \sum_{\sigma} \frac{\widehat{\Sigma}_{4-p_{1}}^{\mu_{\sigma_{p_{1}}}}}{4-p_{1}} \frac{\widehat{\Sigma}_{5-p_{2}}^{\mu_{\sigma_{p_{2}}}}}{5-p_{2}} \frac{\widehat{\Sigma}_{0}^{\mu_{I \backslash \cup_{s=1}^{2}} \sigma_{p_{s}}}}{3} .
$$

Для упрощения вычислений заметим, что имеют место соотношения

$$
\left.(x-y)^{\nu} F_{\nu \mu}(x)\right|_{y=x}=0, \quad F_{\mu \mu}(x)=0
$$

и оператор $\widehat{S}_{l}^{\mu \nu \tau \tau}$ симметричен по индексам $\mu$ и $\nu$.

$\mathrm{C}$ учетом последних замечаний ненулевой вклад дают слагаемые с $p_{1}=p_{2}=0$ и $p_{1}=0, p_{2}=3$, поэтому формула (53) приобретает вид

$$
a_{3}(x, x)=\frac{1}{60} \widehat{\Sigma}_{0}^{\mu \mu \tau \tau \nu \nu}+\frac{1}{12}\left(\widehat{\Sigma}_{2}^{\mu \mu \tau} \widehat{\Sigma}_{0}^{\tau \nu \nu}+\widehat{\Sigma}_{2}^{\mu \tau \tau} \widehat{\Sigma}_{0}^{\mu \nu \nu}\right) .
$$


Из этого выражения и формулы (44) следует, что

$$
\begin{aligned}
& \widehat{\Sigma}_{0}^{\mu \mu \tau \tau \nu \nu}=\frac{4}{3} \nabla_{\mu} F_{\tau \nu}(x) \nabla_{\mu} F_{\tau \nu}(x)+\frac{2}{3} \nabla_{\mu} F_{\mu \nu}(x) \nabla_{\tau} F_{\tau \nu}(x)+ \\
& \quad+\frac{2}{3} F_{\tau \nu}(x) \nabla_{\mu} \nabla_{\mu} F_{\tau \nu}(x)+\frac{4}{3} \nabla_{\mu} \nabla_{\mu} F_{\tau \nu}(x) F_{\tau \nu}(x)-2 F_{\tau \mu}(x) F_{\mu \nu}(x) F_{\nu \tau}(x)
\end{aligned}
$$

И

$$
\widehat{\Sigma}_{2}^{\mu \mu \tau} \widehat{\Sigma}_{0}^{\tau \nu \nu}+\widehat{\Sigma}_{2}^{\mu \tau \tau} \widehat{\Sigma}_{0}^{\mu \nu \nu}=-\frac{1}{15} \nabla_{\mu} F_{\mu \nu}(x) \nabla_{\tau} F_{\tau \nu}(x) .
$$

Таким образом, после подстановки выражений (56) и (57) в (55) окончательная формула записывается как

$$
\begin{aligned}
a_{3}(x, x)= & \frac{1}{45} \nabla_{\mu} F_{\tau \nu}(x) \nabla_{\mu} F_{\tau \nu}(x)+\frac{1}{180} \nabla_{\mu} F_{\mu \nu}(x) \nabla_{\tau} F_{\tau \nu}(x)+ \\
& +\frac{1}{90} F_{\tau \nu}(x) \nabla_{\mu} \nabla_{\mu} F_{\tau \nu}(x)+\frac{1}{45} \nabla_{\mu} \nabla_{\mu} F_{\tau \nu}(x) F_{\tau \nu}(x)-\frac{1}{30} F_{\tau \mu}(x) F_{\mu \nu}(x) F_{\nu \tau}(x)
\end{aligned}
$$

отсюда после взятия следа и интегрирования получается стандартный ответ.

\section{Обратная Р-экспонента}

ПРИЛОЖКНИЕ

Пусть $\chi_{x_{1} \geqslant \cdots \geqslant x_{k}}-$ индикаторная функция множества

$$
\left\{\left(x_{1}, \ldots, x_{k}\right) \in \mathbb{R}^{k}: x_{1} \geqslant \cdots \geqslant x_{k}\right\} \quad \text { при } \quad k>1
$$

и $\chi_{x_{1} \geqslant \cdots \geqslant x_{k}} \equiv 1$ при $k=1$. Пусть функция § задается формулой

$$
\aleph\left(x_{1}, \ldots, x_{k}\right)=\sum_{n=1}^{k} \sum_{k_{1}+\cdots+k_{n}=k}(-1)^{n} \chi_{x_{1} \geqslant \cdots \geqslant x_{k}} \cdots \chi_{x_{k_{1}+\cdots+k_{n-1}+1} \geqslant \cdots \geqslant x_{k_{n}}} .
$$

Используя метод математической индукции, можно доказать следующие леммы.

ЛЕмма 1. Пусть $c_{n}\left(x_{1}, \ldots, x_{n}\right)=\chi_{x_{1} \geqslant \cdots \geqslant x_{n}}$ npu $n \geqslant 1$ u $c_{0}=1$ u nрu $n \geqslant 1$ выполнены соотношения

$$
d_{n}\left(x_{1}, \ldots, x_{n}\right)=-c_{n}\left(x_{1}, \ldots, x_{n}\right)-\sum_{k=1}^{n-1} d_{n-k}\left(x_{1}, \ldots, x_{n-k}\right) c_{k}\left(x_{n-k+1}, \ldots, x_{n}\right) .
$$

Тогда $d_{n}\left(x_{1}, \ldots, x_{n}\right)=\aleph\left(x_{1}, \ldots, x_{n}\right)$ для $n \geqslant 1$.

Лемма 2. Справедливо равенство $\left(x_{1}, \ldots, x_{n}\right)=(-1)^{n} \chi_{x_{1} \leqslant \cdots \leqslant x_{n}}$.

ДокАзАтЕльство. Для $k=1$ формула очевидна. Пусть она верна для $n=k-1$, тогда для $n=k$

$$
\aleph\left(x_{1}, \ldots, x_{n}\right)=-\left(\sum_{k=1}^{n}(-1)^{n-k} \chi_{x_{1} \leqslant \cdots \leqslant x_{n-k}} \chi_{x_{n-k+1} \geqslant \cdots \geqslant x_{n}}\right) .
$$

Ho

$$
\chi_{y_{1} \leqslant \cdots \leqslant y_{n-1}} \chi_{y_{n}}-\chi_{y_{1} \leqslant \cdots \leqslant y_{n-2}} \chi_{y_{n-1} \geqslant y_{n}}=\chi_{y_{1} \leqslant \cdots \leqslant y_{n}}-\chi_{y_{1} \leqslant \cdots \leqslant y_{n-2}} \chi_{y_{n-2} \geqslant \cdots \geqslant y_{n}}
$$


и при $2 \leqslant j \leqslant n-1$

$$
\chi_{y_{1} \leqslant \cdots \leqslant y_{n-j}} \chi_{y_{n-j} \geqslant \cdots \geqslant y_{n}}-\chi_{y_{1} \leqslant \cdots \leqslant y_{n-j-1}} \chi_{y_{n-j} \geqslant \cdots \geqslant y_{n}}=\chi_{y_{1} \leqslant \cdots \leqslant y_{n-j-1}} \chi_{y_{n-j-1} \geqslant \ldots \geqslant y_{n}}
$$

что завершает доказательство.

Для нахождения обратного оператора можно рассмотреть (12) как ряд по фоновому полю. Действительно, после преобразования $B_{\mu}(y) \rightarrow p B_{\mu}(y)$, где $p$ - некоторая варьируемая величина, оператор разлагается в ряд:

$$
\Phi(x, y)=1+\sum_{n=1}^{\infty} p^{n} a_{n}, \quad a_{n}=a_{n}(B, x, y)
$$

Аналогичный вид имеет анзац для обратного оператора:

$$
\Phi^{-1}(x, y)=1+\sum_{n=1}^{\infty} p^{n} b_{n}, \quad b_{n}=b_{n}(B, x, y) .
$$

Из равенства $\Phi^{-1}(x, y) \Phi(x, y)=1$ следует система рекуррентных соотношений

$$
b_{n}=-a_{n}-\sum_{k=1}^{n-1} b_{n-k} a_{k}, \quad n \geqslant 1
$$

откуда с учетом определения 1 и лемм 1 и 2 вытекает, что $\Phi^{-1}(x, y)=\Phi(y, x)$.

Благодарности. Автор выражает благодарность С. Э. Деркачёву, Т. А. Болохову, Д. В. Василевичу и Н. В. Харук за обсуждение и ценные комментарии.

\section{Список литературы}

[1] В. А. Фок, "Собственное время в классической и квантовой механике", Изв. АН СССР. Сер. физ., 4-5 (1937), 551-568.

[2] J. Schwinger, "On gauge invariance and vacuum polarization", Phys. Rev., 82:5 (1951), 664-679.

[3] B. S. DeWitt, "Quantum theory of gravity. I. The canonical theory", Phys. Rev., 160:5 (1967), 1113-1148; "Quantum theory of gravity. II. The manifestly covariant theory", 162:5 (1967), 1195-1239; "Quantum theory of gravity. III. Applications of the covariant theory", 1239-1256.

[4] B. Iochum, C. Levy, D. Vassilevich, "Spectral action beyond the weak-field approximation", Commun. Math. Phys., 316:3 (2012), 595-613, arXiv: 1108.3749.

[5] I. Jack, H. Osborn, "Two-loop background field calculations for arbitrary background fields", Nucl. Phys. B, 207:3 (1982), 474-504.

[6] J.P. Bornsen, A.E. M. van de Ven, "Three-loop Yang-Mills $\beta$-function via the covariant background field method", Nucl. Phys. B, 657:1-3 (2003), 257-303, arXiv: hep-th/0211246.

[7] H. P. McKean, I. M. Singer, "Curvature and the eigenvalues of the Laplacian", J. Differential Geom., 1:1-2 (1967), 43-69.

[8] P. Gilkey, "The spectral geometry of a Riemannian manifold", J. Differential Geom., 10:4 (1975), 601-618.

[9] P. Amsterdamski, A. L. Berkin, D. J. O'Connor, “ $b_{8}$ 'Hamidew' coefficient for a scalar field”, Class. Quant. Grav., 6:12 (1989), 1981-1991. 
[10] I. G. Avramidi, "The covariant technique for the calculation of the heat kernel asymptotic expansion", Phys. Lett. B, 238:1 (1990), 92-97; "A covariant technique for the calculation of the one-loop effective action", Nucl. Phys. B, 355:3 (1991), 712-754.

[11] A. E. M. van de Ven, "Index-free heat kernel coefficients", Class. Quant. Grav., 15:8 (1998), 2311-2344, arXiv: hep-th/9708152.

[12] D. V. Vassilevich, "Heat kernel expansion: user's manual", Phys. Rep., 388:5-6 (2003), 279-360, arXiv: hep-th/0306138.

[13] A. V. Ivanov, "About renormalization of the Yang-Mills theory and the approach to calculation of the heat kernel", EPJ Web Conf., 158 (2017), 07004, 5 pp.

[14] A. O. Barvinsky, G. A. Vilkovisky, "Beyond the Schwinger-DeWitt technique: converting loops into trees and in-in currents", Nucl. Phys. B, 282:1 (1987), 163-188; "Covariant perturbation theory (II). Second order in the curvature. General algorithms", 333:2 (1990), 471-511; "Covariant perturbation theory (III). Spectral representations of the third-order form factors", $512-524$.

[15] I. G. Avramidi, "Heat kernel on homogeneous bundles over symmetric spaces", Commun. Math. Phys., 288:3 (2009), 963-1006, arXiv: math/0701489.

[16] L.D. Faddeev, "Mass in quantum Yang-Mills theory (comment on a Clay Millennium Problem)", Perspectives in Analysis. Essays in Honor of Lenart Carleson's 75th Birthday, Mathematical Physics Studies, 27, eds. M. Benedicks, P. W. Jones, S. Smirnov, B. Winckler, Springer, Berlin, 2011, 63-72.

[17] С. Э. Деркачёв, А. В. Иванов, Л. Д. Фаддеев, "Сценарий для перенормировки в квантовой теории Янга-Миллса в четырехмерном пространстве-времени", ТМФ, 192:2 (2017), $227-234$.

[18] G. M. Shore, "Symmetry restoration and the background field method in gauge theories", Ann. Phys., 137:2 (1981), 262-305.

Поступила в редакцию 24.11.2017, после доработки 24.11.2017, принята к печати 23.04.2018 